reflect the inappropriateness of the control group.

In the Rey Auditory Verbal Learning Test, significantly lower scores on lists A1-A5 were taken to infer a reduction in verbal memory. However, there was no difference between patients and controls for lists A6 and A7. The percentage of words retained between trials A5 and A7 would provide a purer index of retention (Thompson et al, 2005) and would help to better interpret the data.

In the future, meta-analyses of existing data and studies involving assessment of cognitive function and neuroimaging in euthymic patients with bipolar disorder should help elucidate a profile of cognitive deficits and their underlying neurobiological bases.

Chen, E. Y., Shapleske, J., Luque, R., et al (1995) The Cambridge Neurological Inventory: a clinical instrument for assessment of soft neurological signs in psychiatric patients. Psychiatry Research, 56, 183-204.

Ferrier, I. N. \& Thompson, J. M. (2002) Cognitive impairment in bipolar affective disorder: implications for the bipolar diathesis. British Journal of Psychiatry, $\mathbf{1 8 0}$, 293-295.

Goswami, U., Sharma, A., Khastigir, U., et al (2006) Neuropsychological dysfunction, soft neurological signs and social disability in euthymic patients with bipolar disorder. British Journal of Psychiatry, 188, 366-373.

Thompson, J. M., Gallagher, P., Hughes, J. H., et al (2005) Neurocognitive impairment in euthymic patients with bipolar affective disorder. British Journal of

Psychiatry, 186, 32-40.

R. Bharadwaj Department of Psychiatry, Postgraduate Institute of Medical Education and Research, Chandigarh 160012, India.

Email: r_s_bh@yahoo.com

doi: 10.II92/bjp.189.5.468b

Authors' reply Certain aspects of methodology were left out of our paper owing to space constraints. Bharadwaj cites Ferrier \& Thompson (2002) when questioning the exclusion criteria used in our study. Both are co-authors of our paper, which is a result of collaborative research between the Department of Psychiatry in New Delhi and Newcastle since 1998. Whenever possible, similar tests and criteria for euthymia have been used in both centres with occasional variations to respect cultural differences. Use of spouses and siblings as members of the control group was acceptable, as it brought together people of broadly similar backgrounds. Although this might have resulted in the inclusion of a limited number of controls who were at risk of developing bipolar disorder, it minimised differences between people with bipolar disorder and controls without greatly confounding our results.

For verification of euthymia participants were seen at least twice, separated by a minimum of 1 month, before they were tested. Clinical judgement of euthymia was reinforced by a Hamilton Rating Scale for Depression score $<8$ and a score $<20$ on Bech's modification of Beigel's Manic State Rating Scale on both occasions. The stability of mood during the in tervening period was assessed clinically on a weekly basis. We were not aware of any Hindi version of the Structured Clinical Interview for DSM-III - patient version. The exclusion of other psychiatric morbidity was based on clinical interviews by two highly experienced psychiatrists, complemented by careful mapping of life charts using the techniques of Post et al (1998).

Soft neurological signs were assessed with the widely used modified Kolakowska battery. We are unsure whether the use of other batteries, such as the Cambridge Neurological Inventory, would substantially alter our findings. Involving a second rater would perhaps increase reliability but would extend the assessment time unreasonably.

Not surprisingly, soft signs were found in the control group, but only at about one-quarter of the severity seen in people with bipolar disorder. The maximum score on the modified Kolakowska battery was 45. The maximum score for controls was 9 whereas the mean for patients was 13.9 . Control scores mainly comprised minimum scores on a few of the 15 items. In a subsequent article (further details available on request) we report high levels of soft signs in the youngest patients with bipolar disorder. There is no evidence that soft signs progress with age in bipolar disorder, whereas in controls there is significant $(P<0.01)$ progression with age.

List A7 of the Rey Auditory Verbal Learning Test measures retention after $20 \mathrm{~min}$. We have further analysed these data and found no difference between the groups.

We agree that 'duration of illness'; actually describes 'duration of illness episodes'. The actual mean duration of illness was 9.1 years $($ s.d. $=6.0)$. Data concerning marital status and occupation were collected but were omitted for brevity. We did not wish to control for handedness or birth injuries as potential confounders as we regarded these differences to be part of the spectrum of people with bipolar disorder. We did not include those who had recently received electroconvulsive therapy ( $\geqslant 6$ months). Finally, we would agree that there is a need for meta-analyses and have recently published such a study (Robinson et al, 2006).

Ferrier, I. N. \& Thompson, J. M. (2002) Cognitive impairment in bipolar affective disorder: implications for the bipolar diathesis. British Journal of Psychiatry, $\mathbf{1 8 0}$ 293-295

Post, R. M., Roy-Byrne, P. P. \& Uhde, T. W. (1998) Graphic representation of the life course of illness in patients with affective disorder. American Journal of Psychiatry, 145, 844-848.

Robinson, L. J., Thompson, J. M., Gallagher, P., et al (2006) A meta-analysis of cognitive deficits in euthymic patients with bipolar disorder. Journal of Affective Disorders, 93, 105-115.

U. Goswami Department of Psychiatry, West Park Hospital, Darlington, UK

P. B. Moore Department of Psychiatry, Royal Victoria Infirmary, Newcastle upon Tyne NEI 4LP,UK. Email: pbrianm@aol.com

doi: $10.1192 /$ bjp.189.5.469

\section{Delay in onset of action of antidepressants}

In an important editorial, Mitchell (2006) marshalls evidence to show that we do not have to wait 2 weeks for antidepressants to work.

Why has it been so difficult so far to show that they work in the first few days? In addition to the reasons that Mitchell sets out, I should like to mention a further problem. If you analyse the results on a day-by-day basis, it is hard to obtain sufficient statistical power to distinguish the early response to the drug from the response to the placebo, since you have just the scores for that day.

In 1996 my colleagues and I published evidence that the fall in scores on the Hamilton Rating Scale for Depression followed an exponential decay curve with a correlation coefficient of 0.99 (Priest et al, 1996; Livingston \& Clark, 1997). This observation corresponds with Mitchell's remarks on the steep fall in scores in the first 2 weeks. A comparison of the slope of the curve for the active drug with the placebo, using all of the data, gives a very sensitive way of testing for efficacy.

By plotting the log of the depression scores against time, a straight line is obtained. Thus the recovery from depression 